\title{
Erratum: Intersubband plasmon excitations in doped carbon nanotubes [Phys. Rev. B 99, 075403 (2019)]
}

\author{
Daria Satco, Ahmad R. T. Nugraha • 1 , M. Shoufie Ukhtary, Daria Kopylova, \\ Albert G. Nasibulin, and Riichiro Saito
}

(Received 29 July 2021; published 7 September 2021)

DOI: 10.1103/PhysRevB.104.119904

The paper contains some errors that should be corrected as outlined below. All the calculated results and conclusions are unaffected by the corrections.

(1) In Eq. (6), the imaginary number $i$ was missing in the denominator of the conductivity prefactor. The correct expression should read

$$
\sigma_{\perp}^{i j}=\frac{16}{i d_{t}} \frac{e^{2}}{h}\left(\frac{\hbar^{2}}{m}\right)^{2} \int_{-\pi / T}^{\pi / T} \frac{d k}{2 \pi}\left|\mathcal{M}_{i j}^{\perp}(k)\right|^{2} \frac{f\left[E_{i}(k)\right]-f\left[E_{j}(k)\right]}{E_{j}(k)-E_{i}(k)-\hbar \omega+i \Gamma} \frac{1}{E_{j}(k)-E_{i}(k)} .
$$

(2) In Appendix A, before Eq. (A13), the sentence "For the light propagating parallel to the nanotube axis (n $\|$ T) ..." should read "For the light with polarization parallel to the nanotube axis $(\mathbf{n} \| \mathbf{T})$...".

(3) If we follow Fig. 9(b) properly, the unit vector $\mathbf{n}_{\perp}$ for the light polarization perpendicular to the tube axis should be defined as

$$
\mathbf{n}_{\perp}=\sin \theta_{j} \mathbf{e}_{C}+\cos \theta_{j} \mathbf{e}_{r},
$$

where $\mathbf{e}_{C}\left(\mathbf{e}_{r}\right)$ is a unit vector tangential (normal) to the tube surface. The unit vector $\mathbf{e}_{C}$ has been defined in Eq. (A17), while $\mathbf{e}_{r}$ was not given in the paper because only the tangential component of the vector potential determines the optical absorption. With this consideration, we wrote an incorrect definition of the vector potential for the perpendicular polarization in Eq. (A14) of the original paper:

$$
\mathbf{A}_{\mathbf{q}}^{\perp}[\mathbf{R}(j)]=A_{0} \cos \theta_{j} \mathbf{n}_{\perp} e^{i \mathbf{q} \cdot \mathbf{R}(j)},
$$

which should read

$$
\mathbf{A}_{\mathbf{q}}^{\perp}[\mathbf{R}(j)]=A_{0} \sin \theta_{j} \mathbf{e}_{C} e^{i \mathbf{q} \cdot \mathbf{R}(j)}=\frac{A_{0}}{2 i} \mathbf{e}_{C}\left(e^{i \theta_{j}}-e^{-i \theta_{j}}\right)\left(1+i q \frac{d_{t}}{2} \sin \theta_{j}\right) .
$$

Using Eq. (A14) as corrected above, the optical matrix elements for the perpendicular polarization given in Eq. (A16) should read

$$
\begin{aligned}
\left\langle s_{2}, \mu_{2}, k_{2}\left|\mathbf{A}_{\mathbf{q}} \cdot \nabla\right| s_{1}, \mu_{1}, k_{1}\right\rangle= & \frac{A_{0}}{N} \sum_{\ell, \ell^{\prime}=A, B} C_{k_{2} \mu_{2} \ell^{\prime}}^{s_{2} *} C_{k_{1} \mu_{1} \ell}^{s_{1}} \sum_{j} \frac{1}{2 i}\left(e^{i\left[\left(\mathbf{k}_{1}-\mathbf{k}_{2}\right) \cdot \mathbf{R}(j)+\theta_{j}\right]}-e^{i\left[\left(\mathbf{k}_{1}-\mathbf{k}_{2}\right) \cdot \mathbf{R}(j)-\theta_{j}\right]}\right) \\
& \times \sum_{j^{\prime}} \mathbf{e}_{C} \cdot\left\langle j^{\prime}, \ell^{\prime}|\nabla| j, \ell\right\rangle e^{-i \mathbf{k}_{2} \cdot\left[\mathbf{R}\left(j^{\prime}\right)-\mathbf{R}(j)\right]},
\end{aligned}
$$

and Eq. (A20) should read

$$
\begin{aligned}
\left\langle s_{2}, \mu_{2}, k_{2}\left|\mathbf{A}_{\mathbf{q}} \cdot \nabla\right| s_{1}, \mu_{1}, k_{1}\right\rangle= & A_{0} \sum_{\ell, \ell^{\prime}=A, B} C_{k_{2} \mu_{2} \ell^{\prime}}^{s_{2} *} C_{k_{1} \mu_{1} \ell}^{s_{1}} \delta\left(k_{1}-k_{2}\right) \frac{1}{2 i}\left[\delta\left(\mu_{1}-\mu_{2}+1\right)-\delta\left(\mu_{1}-\mu_{2}-1\right)\right] \\
& \times \sum_{j} \mathbf{e}_{C} \cdot\left\langle j, \ell^{\prime}|\nabla| 0, \ell\right\rangle e^{-i \mathbf{k}_{2} \cdot \mathbf{R}(j)}
\end{aligned}
$$

The use of $A_{0} \sin \theta_{j}$ in the corrected Eq. (A14), instead of $A_{0} \cos \theta_{j}$ in the original Eq. (A14), does not alter the optical selection rule because the selection rule is determined only by $\theta_{j}$. The absorption spectra are not affected either since we calculated the absorption spectra from the squared absolute value of the matrix element for each selection rule of $\mu_{1}$ and $\mu_{2}$. Therefore, all the calculated absorption spectra in the paper remain valid. 
It should be noted that for the case of parallel polarization, since the vector potential lies in the direction of the tube axis, we will obtain $\mathbf{n}_{\|}=\mathbf{e}_{T}$, where $\mathbf{e}_{T}$ is the unit vector in the direction of tube axis as defined in Eq. (A17) of the paper. Thus, Eqs. (A21) and (A22) are correct.

(4) The value of Fermi energy $E_{F}$ in the caption of Fig. 10(b) should read $2.5 \mathrm{eV}$.

The authors thank Dr. Sake Wang (a visiting scientist at Tohoku University) who pointed out the errors when he rederived all formulas in the paper for applying our computer program (Ref. [48] of the paper) to a new project. He confirms that he can reproduce all the original calculated results by fixing and rerunning the program. 